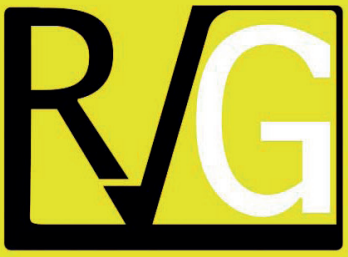

Julio - Septiembre, 2021

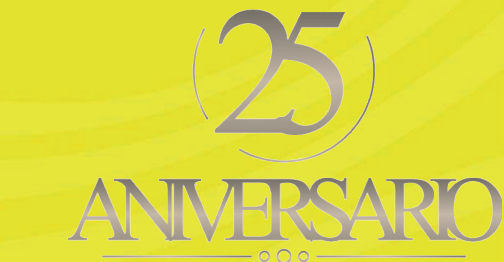

ANMERSARO
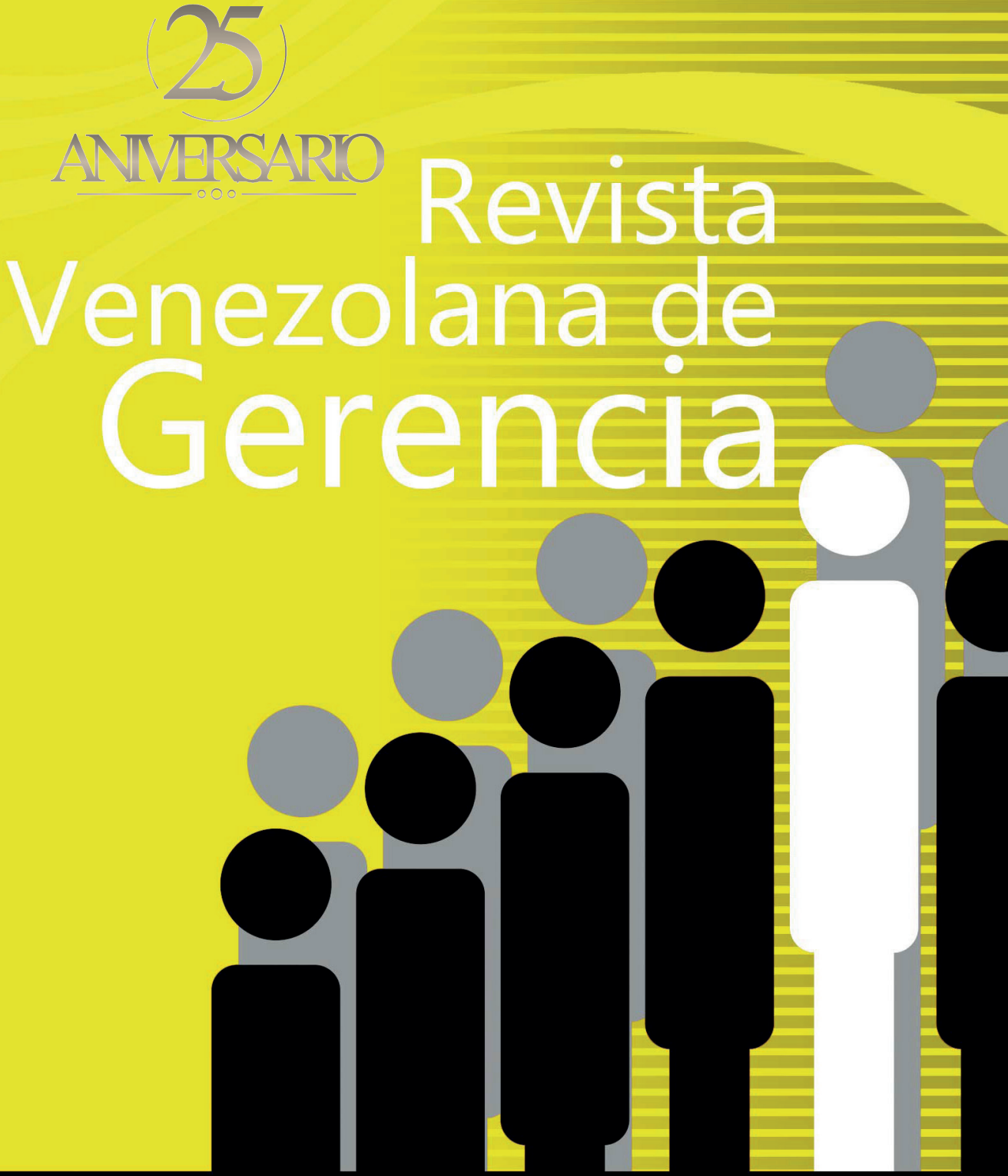

UNIVERSIDAD DEL ZULIA (LUZ)

Facultad de Ciencias Económicas y Sociales Centro de Estudios de la Empresa

ISSN 1315-99

Esta obra está bajo una licencia de Creative Comm Reconocimiento-NoComercial-Compartirlgual 3.0 Unpo http://creativecommons.org/licenses/by-nc-sa/3.0/deed.es 
COMO CITAR: López Armes, E. (2021). Responsabilidad social y el compromiso organizacional de empleados públicos del Perú. Revista Venezolana de Gerencia (RVG), 26(95), 656-668. https://doi. org/10.52080/rvgluz.27.95.14

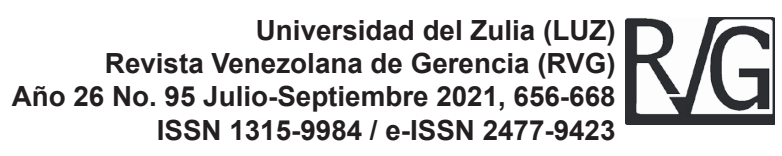

Universidad del Zulia (LUZ)

e-ISSN $2477-9423$

\title{
Responsabilidad social y el compromiso organizacional de empleados públicos del Perú
}

\author{
López Armes, Edy Luisa*
}

\section{Resumen}

La responsabilidad social y el compromiso organizacional son pilares de toda institución pública; permiten gestionar, de manera sostenible y ética sus relaciones con el ámbito interno y externo, permitiendo brindar buenos resultados en la gestión municipal y por ende lograr la satisfacción de los empleados. El estudio tiene como objetivo central determinar la relación entre la responsabilidad social y el compromiso organizacional de empleados públicos de la Municipalidad Provincial de Huancayo en el Perú. Se cotejó la información obtenida de un cuestionario aplicado a una muestra de 186 empleados elegidos al azar, entre varones y mujeres (autoridades, funcionarios y personal administrativo). Los resultados indican que no existe correlación significativa entre la responsabilidad social y el compromiso organizacional $(r=0.098, p=0.182)$. Se concluye que los trabajadores públicos desconocen y tienen indiferencia hacia la responsabilidad social, es decir, no hay capacidad de respuesta de la Municipalidad Provincial de Huancayo hacia sus grupos de interés. Además, respecto al compromiso organizacional; los servidores públicos no se identifican y no tienen ningún nivel de integración con la institución, no toman conciencia de la importancia de ciertos aspectos que lo llevan a considerar su permanencia o continuidad en la organización y finalmente, no se sienten obligados a permanecer como empleados en la organización.

Palabras clave: Responsabilidad social; compromiso organizacional; administración pública; grupos de interés.

* Cursando el Doctorado en Educación, Universidad Nacional del Centro del Perú; Magister en comunicación, Universidad Nacional del Centro del Perú; docente en la Universidad Nacional del Centro, Unidad Académica de Estudios Generales: elopez@uncp.edu.pe. ORCID: https://orcid.org/0000-0002-2170-0298 


\title{
Social responsibility and the organizational commitment of public employees of Perú
}

\begin{abstract}
Social responsibility and organizational commitment are pillars of every public institution; They allow to manage, in a sustainable and ethical way, its relations with the internal and external environment, allowing to provide good results in municipal management and therefore achieve employee satisfaction. The main objective of the study is to determine the relationship between social responsibility and the organizational commitment of public employees of the Provincial Municipality of Huancayo in Peru. The information obtained from a questionnaire applied to a sample of 186 employees chosen at random, between men and women (authorities, officials and administrative personnel) was collated. The results indicate that there is no significant correlation between social responsibility and organizational commitment $(r=0.098, p=0.182)$. It is concluded that public workers are unaware of and have indifference towards social responsibility, that is, there is no response capacity of the Provincial Municipality of Huancayo towards its interest groups. In addition, regarding the organizational commitment; Public servants do not identify themselves and do not have any level of integration with the institution, they do not become aware of the importance of certain aspects that lead them to consider their permanence or continuity in the organization and finally, they do not feel obliged to remain as employees in the organization.
\end{abstract}

Keywords: Social responsibility; organizational commitment; public administration; stakeholders.

\section{Introducción}

La responsabilidad social (RS) proporciona una interesante oportunidad para que las instituciones del estado puedan gestionar sustentable y éticamente sus relaciones en el entorno interno y externo. La RS es un término aplicable a cualquier tipo de organización, independientemente del sector económico al que pertenezca (Instituto de Normas Técnicas de Costa Rica, INTECO, 2010), su tamaño y/o estructura, dejando atrás el mito de que se limita a acciones exclusivas de empresas privadas. $Y$ estas a su vez deben ser asumida con voluntad $y$ transparencia por la gestión pública y para ello la aplicación social corporativa puede contribuir de manera considerable al fortalecimiento de la democracia participativa (Gaete, 2008:56).

La aplicación de la responsabilidad social en la gestión pública implica que los servidores públicos deben trabajar articuladamente con otras instituciones estatales y organismos privados, pero ocurre todo lo contrario, la función que desempeñan es muchas veces de carácter privativo y monopólico, lo cual fomenta una cultura de aislamiento y descoordinación, duplicando en varias oportunidades, los esfuerzos y recursos innecesariamente, y generando la 
insatisfacción de los ciudadanos.

Según Canyelles (2011:77), se debe implementar la introducción de cláusulas sociales, la elaboración de códigos éticos y la publicación de memorias de sostenibilidad, las cuales se verán reflejadas en la mejora de la gobernanza, la eficacia y la eficiencia de las políticas públicas, la participación, el diálogo con los grupos de interés, la transparencia, la legitimidad, el desarrollo de territorios socialmente responsables o la misma formalización de la gestión de la RS.

Del mismo modo, la aplicación de la RS en el ámbito público responde satisfactoriamente cuando se logra una transversalidad entre las aspiraciones y demandas de la ciudadanía (consideración de los inmigrantes, discapacitados, no a la discriminación, la equidad, la diversidad, el empoderamiento o la participación), las cuales deben ser incorporadas como variables a los procesos de gestión pública. Es por ello que este trabajo considera el quehacer de las municipalidades en particular como parte de su objeto de estudio.

La RS es un tema relativamente nuevo en el Perú, no tienen más de doce años. Su aplicación se estuvo dando en las empresas propiamente dichas. Al inicio se pensó que se trataba de una moda para tranquilizar las "culpas" de estas, para ello, había que devolver algo de lo recibido e invertir en la sociedad. Sin embargo, habiendo pasado ya más de una década desde que se introdujera este término, no hay duda de que el tema va en serio.

Recientemente, la Municipalidad Distrital de La Victoria en el Perú, (2019) publicó la Ordenanza $\mathrm{N}^{\circ}$ 006-2019-CPPAL-CDSMC/MDLV, que busca incentivar a las personas naturales o jurídicas, nacionales o extranjeras, con o sin fines de lucro, a desarrollar actividades de responsabilidad social en ese distrito; comprometiéndose de manera voluntaria a asumir un comportamiento que contribuya al desarrollo sostenible de La Victoria mediante una participación activa dentro de una cultura que hace posible el buen vivir y la convivencia armónica; y resulta pertinente propiciar dichos mecanismos de participación social para asegurar una permanente cooperación entre la población y la autoridad municipal.

La Municipalidad Provincial de Huancayo en el Perú representa a los pobladores del lugar y promueve el desarrollo de la ciudad en coordinación con el Gobierno Regional de Junín y el Gobierno nacional para facilitar la competitividad y propiciar mejores condiciones de vida de su población.

En ese contexto, el compromiso organizacional es un factor muy importante inherente al desempeño de los empleados públicos de la Municipalidad, ya que un buen nivel del mismo, asegura la satisfacción y buenos resultados para la organización. Por ello, se traza como objetivo central de la investigación determinar la relación entre la responsabilidad social y el compromiso organizacional de empleados públicos de la Municipalidad Provincial de Huancayo en el Perú.

La presente investigación es de carácter descriptivo correlacional, de acuerdo con (Sánchez et al. 2018:80); descriptiva porque identifica, describe y analiza las características de la responsabilidad social y el compromiso organizacional de los empleados públicos de la Municipalidad Provincial de Huancayo, y correlacional porque se quiere establecer el grado de relación estadística que existe entre las variables. 


\section{Responsabilidad social: consideraciones teoricas}

\section{Existen varias teorías relacionadas}

a la responsabilidad social, e incluso continúa en constante crecimiento. Desde su aparición tuvo diversas denominaciones, aunque las más utilizadas son responsabilidad social corporativa (RSC) y responsabilidad social empresarial (RSE).

Desde un principio, en España, se asumió la RSC como traducción literal de inglés (Corporate Social Responsibility), debido básicamente a la necesidad de su aplicación inmediata y a la falta de una base conceptual sólida (Asociación Española de Contabilidad y Administración, AECA, 2006). A partir de esta primera dificultad, de orden etimológico, investigadores $y$ empresarios comienzan a asumir sus propios conceptos, de manera que se da inicio a un dispendioso proceso de asociaciones terminológicas.

En los años ochenta se inicia a defender la idea de la contribución al Estado del bienestar y la calidad de vida de los ciudadanos y es así que surge a nivel internacional el interés de los gobernantes de incorporar dentro de su política pública la economía social y solidaria para asegurar su impacto y eficiencia (Luque et al. 2019:290)

De acuerdo a la Guía ISO 26000 (INTECO, 2010:2), la responsabilidad de una organización ante los impactos que sus decisiones y actividades ocasionan en la sociedad y en el medio ambiente, mediante un comportamiento ético $\mathrm{y}$ transparente que contribuya con el desarrollo sostenible, la salud y el bienestar de la sociedad; toma en cuenta las expectativas de las partes interesadas; cumpla con las leyes aplicables y con las normativa internacionales de comportamiento; está integrada en toda la organización y se lleva a la práctica en todas sus relaciones.

Asimismo, Vargas-Merino (2021) señala que las "organizaciones, tienen como reto la satisfacción combinada o fusionada entre la sociedad y ellas, se trata pues de promover el desarrollo personal yel bienestarcomún, lográndose materializar con la satisfacción de las necesidades sociales". Ferrer (2013) señala que la responsabilidad social "asume compromisos hacia lo interno de la empresa y sus trabajadores, y hacia el entorno en el que se inserta; de esta manera supera las actuaciones tradicionales".

Actualmente en las entidades públicas y privadas del Perú, la responsabilidad social no está regulada por ninguna ley, solo se da de manera voluntaria y es adaptada a su propia filosofía que genera un efecto positivo para los trabajadores considerados parte esencial del entorno interno, así como para los ciudadanos y las comunidades (Artigas, 2012: 25).

Con respecto a los trabajadores en una entidad municipal apuntan a medir su equilibrio permanente en términos de capacitación, trabajo, familia y ocio, contratación no discriminatoria, igualdad de remuneración y perspectivas de carrera. Así lo confirmaría en su investigación (Cano et al. 2016: 123), la responsabilidad social gubernamental, es la capacidad de respuesta que tienen las instituciones gubernamentales hacia sus grupos de interés, a través del diseño y ejecución de políticas públicas, de forma que su responsabilidad hacia las actividades de los ciudadanos, organizaciones y empresas es tanto externa como interna. La responsabilidad social se aplica a los 3 niveles de 
gobierno, central, regional y municipal (Borthiry, 2002:56).

La publicidad en las entidades municipales es una valiosa herramienta para difundir información cuyo objetivo es la acción social, por lo tanto, es de vital importancia que sea realizada con responsabilidad social, contrariamente en un estudio realizado en Argentina por (Tomba et al. 2020:125 ) encontraron que las piezas publicitarias relacionada a las categorías de salud y sociedad es excesivamente genérico, lejos de un enfoque participativo y dialogado con mensajes unidireccionales. Si se consignaran esfuerzos para incorporar estas temáticas en tiempos y espacios de ocio donde lo artístico y recreativo forma un marco efectivo se alcanzaría aumentar el impacto social.

Sobre la base de los conceptos y estrategias de responsabilidad social empresarial desarrollados en el mundo, existen tres grandes modelos de responsabilidad social empresarial, como son: los ambientales (protección al medio ambiente), económicos (valores económicos justos y equilibrados) y sociales (promover los valores éticos y sociales) con los que se busca igualar las prácticas empresariales con sus responsabilidades ante la sociedad. Con lo anterior las organizaciones podrán gozar de un crecimiento económico de una forma sostenible, con labores que fortifiquen el equilibrio ambiental $y$ desarrollo social.

En el Perú se ha generado un modelo de responsabilidad social empresarial (Cornejo, 2014:52). Este incluye la identificación de los principales stakeholders de la empresa, diversas acciones que se pueden realizar para cada uno de estos y los beneficios que generan las mismas. Los stakeholders o grupos de interés son los grupos o individuos relacionados con la empresa que pueden ser afectados por esta e influir en la misma.

Según Canessa y García (2005:41), son siete stakeholders; el accionista/inversionista, los empleados públicos y sus familias, los clientes, los proveedores, el gobierno, la comunidad y el medio ambiente.

Las mismas que se adaptan al ámbito público, grupos de interés interno (alcalde, empleados, conceso municipal) y los grupos de interés externo (gobierno, empresas, junta de vecinos, organizaciones deportivas, organizaciones culturales, organizaciones de salud, organizaciones del adulto mayor, organizaciones juveniles, organizaciones de seguridad, organizaciones sociales). De acuerdo al ámbito social de la Triple Bottom Line, las dimensiones de la responsabilidad social son: valores y principios éticos, protección del ambiente, proveedores, marketing responsable, apoyo a la comunidad y gobierno y sociedad (Castillo, 2007:148)

\section{Compromiso organizacional: ¿de qué hablamos?}

El conocimiento no basta para resolver todos los problemas $y$ alcanzar las metas y objetivos de una organización; también es necesaria una serie de habilidades, destrezas y actitudes. El compromiso organizacional es considerado una de las actitudes más importantes hacia la institución para lograr un beneficio común (López, 2018:45)

Para que una institución pública o privada logre su estabilidad es importante que los servidores públicos se sientan 
realmente comprometidos. En ese sentido, Meyer y Allen (1991) plantean las dimensiones del compromiso organizacional; el compromiso afectivo, se refiere a la identificación y la implicación emocional del individuo con los valores de la organización; compromiso de continuidad, se basa en los costes personales que supondría para el individuo al abandonar la organización y el compromiso normativo, se entiende como la obligación moral que sienten los trabajadores de permanecer en la institución.

Según, Omar y Florencia (2008), existen algunos factores determinantes del compromiso afectivo: Los empleados estarán más satisfechos cuando pueden satisfacer sus propias necesidades, porque las condiciones del trabajo son adecuadas para ello. Asimismo, los autores indican que los factores determinantes del compromiso y del abandono son: El conflicto, la ambigüedad del rol, las relaciones interpersonales deficientes, clima laboral desfavorable. Mientras que los factores personales determinantes del compromiso: la edad, la educación, el género, el tiempo de permanencia en la organización, el puesto de trabajo, el nivel jerárquico dentro de la organización.

\section{Correlación de la responsabilidad social y compromiso organizacional}

La Municipalidad Provincial de Huancayo está ubicada en la capital de la región de Junín, en el centro de Perú. Con una población de 511035 habitantes (INEI, 2017:27).

La provincia de Huancayo es una de las nueve provincias que conforman el departamento de Junín, bajo la administración del gobierno regional de Junín y se ubica en la parte suroeste, sobre los LS $12^{\circ} 03^{\prime} 51 \|$ de latitud y LS $75^{\circ} 12^{\prime} 30 \| l$ de longitud con referencia al meridiano de Greenwich. Ubicado sobre 3249 m.s.n.m. a una distancia de 299 Km. de la ciudad de Lima, capital de la República del Perú. La fortaleza mayor del estrato alto es el sector comercio, dinámicamente articulada con la economía regional de la parte central del Perú, mientras que sus principales debilidades son su falta de propagación en los distritos y su carencia de liderazgo para lograr la universalización de la economía de mercado y la extinción de las formas atrasadas que constituyen nichos de pobreza y extrema pobreza, a pesar de la disminución de ambas en los últimos años.

Desde la perspectiva metodológica, el estudio precisa variables como la responsabilidad social y el compromiso organizacional, con una población de 646 empleados públicos (autoridades, funcionarios y personal administrativo) y una muestra de 186 entre varones (114) y mujeres (72); de las edades entre 21-33 años (78), 3445 años (64), 4657 años (33) y 58-66 años (11); el tipo de contrato: D.L.N. ${ }^{\circ}$ 1024 (5), D.L. N. 276 - Empleados (49), D.L. N. ${ }^{\circ}$ 1057-Contratados CAS (126) y Dietas (6); de acuerdo al cargo: autoridades (6), funcionarios (10) y personal administrativo (170); según el nivel de instrucción: secundaria (45) y superior (141); asimismo, en relación al estado civil: soltero (76), casado (65), conviviente (40), divorciado (5), y por el tiempo de servicio: nuevos (158), medianos (18), antiguos (10), utilizando un margen de error del $5 \%$.

Para la confiabilidad de los instrumentos se calculó mediante la medida de estabilidad y se encontró 
el valor del alfa de Cronbach para la responsabilidad social: $(\alpha=0.955)$ y para el compromiso organizacional: $(\alpha=0.815)$ y para determinar la validez, se relacionaron a través de ítems, subtest y test con el programa informático SPSS 21, empleando el estadístico $r$ de Pearson corregido, el cual demostró correlaciones altas, por ende, los cuestionarios poseen una alta validez y miden las variable que pretendimos medir. En tanto, el instrumento de responsabilidad social alcanzó un valor máximo de 0.642 y mínimo de 0.228 ; mientras que, para el compromiso organizacional, el valor máximo fue de 0.612 y el mínimo 0.226 .

Para la recolección de datos se utilizó la técnica de la encuesta y el instrumento el cuestionario. En la variable responsabilidad social se aplicaron 72 ítems en escala de tipo Likert con seis dimensiones (Valores y principios éticos, protección del ambiente, proveedores, marketing responsable, apoyo a la comunidad, gobierno y sociedad). Y en el compromiso organizacional 18 ítems en escala tipo Likert con tres dimensiones (Compromiso afectivo, compromiso de continuidad y compromiso normativo), tomando como base el instrumento desarrollado en la teoría de Meyer,
Allen y Smith (1991) de 17 preguntas, al cual se le agregó un ítem más en la dimensión compromiso afectivo a fin de darle proporcionalidad al número de ítems por cada dimensión, haciendo un total de 18 preguntas que fueron validadas correctamente.

En el procesamiento de datos, se empleó la prueba no paramétrica de comparación de dos muestras independientes $U$ de Mann Whitney y Prueba de Kruskal - Wallis para comparar y determinar las diferencias entre la responsabilidad social, el compromiso organizacional y las variables demográficas (sexo, nivel de instrucción, edad, tipo de contrato, cargo, estado civil y tiempo de servicio respectivamente). Para determinar la correlación de las variables de estudio y sus dimensiones se aplicó la Rho de Sperman.

Los resultados se muestran en los siguientes párrafos. En la tabla 1 se aprecia los resultados del coeficiente de correlación de Spearman de la responsabilidad social (RS) y el compromiso organizacional (CO) y sus respectivas dimensiones; estos indican que entre las dos variables mencionadas no existe relación significativa $(r=0.098)$. 
Tabla 1

\section{Coeficiente de correlación de Spearman de la responsabilidad social empresarial y el compromiso organizacional y sus dimensiones}

\begin{tabular}{|c|c|c|c|c|c|c|c|c|c|c|c|}
\hline & & VPE & PMA & $\mathrm{P}$ & MR & $A C$ & GS & RS & $\mathrm{CC}$ & $\mathrm{CA}$ & $\mathrm{CN}$ \\
\hline \multirow{2}{*}{$\begin{array}{l}\text { Protección del } \\
\text { medio ambiente } \\
\text { (PMA) }\end{array}$} & $r$ &, $423^{* *}$ & & & & & & & & & \\
\hline & Sig. (bilateral) & ,000 & & & & & & & & & \\
\hline \multirow{2}{*}{ Proveedores $(P)$} & $r$ & ,423** & ,448* & & & & & & & & \\
\hline & Sig. (bilateral) &, 000 &, 000 & & & & & & & & \\
\hline \multirow{2}{*}{$\begin{array}{l}\text { Marketing } \\
\text { responsable (MR) }\end{array}$} & $r$ & ,410** &, $561^{* *}$ & ,430** & & & & & & & \\
\hline & Sig. (bilateral) &, 000 & ,000 &, 000 & & & & & & & \\
\hline \multirow{2}{*}{$\begin{array}{l}\text { Apoyo a la } \\
\text { comunidad (AC) }\end{array}$} & $r$ &, $404^{* *}$ &, $605^{* *}$ &, $386^{* *}$ &, $565^{* *}$ & & & & & & \\
\hline & Sig. (bilateral) & ,000 &, 000 &, 000 &, 000 & & & & & & \\
\hline \multirow{2}{*}{$\begin{array}{l}\text { Gobierno y } \\
\text { sociedad (GS) }\end{array}$} & $r$ &, $289^{* *}$ &, $361^{* *}$ & ,230"* & ,329** &, $540^{* *}$ & & & & & \\
\hline & Sig. (bilateral) &, 000 &, 000 & ,002 &, 000 &, 000 & & & & & \\
\hline \multirow{2}{*}{$\begin{array}{l}\text { Responsabilidad } \\
\text { social (RS) }\end{array}$} & $r$ &, $565^{* *}$ &, $642^{* *}$ & ,450"* &, $617^{* *}$ &, $654^{* *}$ &, $543^{* *}$ & & & & \\
\hline & Sig. (bilateral) &, 000 &, 000 &, 000 &, 000 &, 000 & ,000 & & & & \\
\hline \multirow{2}{*}{$\begin{array}{l}\text { Compromiso } \\
\text { continuo (CC) }\end{array}$} & $r$ &,- 034 & ,141 &, 040 & ,112 & ,082 &, $164^{*}$ & , 102 & & & \\
\hline & Sig. (bilateral) & ,641 &, 056 & ,586 & ,129 &, 264 & ,026 & , 168 & & & \\
\hline \multirow{2}{*}{$\begin{array}{l}\text { Compromiso } \\
\text { afectivo (CA) }\end{array}$} & $r$ & ,112 & 131 &, $148^{*}$ & ,082 &, $148^{*}$ &, $268^{* *}$ & ,142 &, $167^{*}$ & & \\
\hline & Sig. (bilateral) & ,127 &, 074 &, 044 & ,266 &, 044 &, 000 & ,052 & ,023 & & \\
\hline \multirow{2}{*}{$\begin{array}{l}\text { Compromiso } \\
\text { normativo }(\mathrm{CN})\end{array}$} & $r$ &, $165^{*}$ &, $236^{* *}$ & , 109 &, $307^{* *}$ &, $228^{* *}$ &, $273^{* *}$ &, $285^{* *}$ & ,195* &, $410^{* *}$ & \\
\hline & Sig. (bilateral) &, 025 &, 001 & ,138 &, 000 &, 002 &, 000 &, 000 &, 008 &, 000 & \\
\hline \multirow{2}{*}{$\begin{array}{l}\text { Compromiso } \\
\text { organizacional } \\
\text { (CO) }\end{array}$} & $r$ & ,012 & ,101 &, 028 &, 118 &, 084 & ,220** & ,098 & ,418" &, $501^{* *}$ &, $560^{* *}$ \\
\hline & Sig. (bilateral) & ,875 & ,170 & ,702 & , 108 & ,255 & ,003 & ,182 & ,000 & ,000 & ,000 \\
\hline
\end{tabular}

**. La correlación es significativa en el nivel 0,01 (bilateral).

*. La correlación es significativa en el nivel 0,05 (bilateral).

Fuente: Elaboración propia

Estos resultados contrastan con lo que encontraron en sus investigaciones Hernández y Castro, (2015) y Chávez (2019) que existe una alta correlación entre responsabilidad social y compromiso organizacional, debido a que las praxis de la responsabilidad social influyen directamente en el bienestar del trabajador por ende su compromiso organizacional es mayor; evidenciando además que ambas variables van de la mano, debido a que si una organización no está socialmente comprometida con sus trabajadores como punto de partida 
difícilmente lo será de manera externa.

Asimismo, luego de realizar

la correlación entre la variable responsabilidad social y las dimensiones de la variable compromiso organizacional, se aprecia que solo hay una correlación significativa entre la responsabilidad social y el compromiso normativo $r=0.285$. Se entiende que ser responsable socialmente tiene relación con la obligación moral que sienten los servidores públicos de permanecer en la municipalidad, así lo confirma, (Đorđević et al. 2020) los trabajadores sienten lealtad y permanecen cumpliendo sus funciones y metas en función a los valores de la entidad.

Por su parte, Al-Haroon y AlQahtani (2020) en un estudio realizado en un hospital público de Arabia Saudita, encontraron que un gran número de los trabajadores tienen un mayor compromiso organizacional relacionado positivamente con variables sociodemográficas, como la edad y la nacionalidad, y el único predictor positivo fue la edad. Y recomiendan se debe mejorar el compromiso organizativo mediante el desarrollo de estrategias para contratar, atraer $y$ retener trabajadores comprometidos.

Finalmente, se encontró una correlación significativa entre la variable compromiso organizacional y la dimensión de la responsabilidad social, gobierno y sociedad $(r=0.220, p=0.003)$, tabla 1. Esto indica que existe relación entre el compromiso organizacional y las practicas anticorrupción y coima, liderazgo e influencia social. Contrariamente se observó en la praxis varias denuncias de delitos contra la administración pública como: delito de peculado malversación de fondos públicos por el caso del Parque de Constitución de la ciudad de Huancayo
(La Republica, 28/05/2019: política), asimismo, la Contraloría general detectó irregularidades en el proceso de selección para la ejecución del proyecto 'Mejoramiento de servicios turísticos públicos de la Plaza Constitución', en la ciudad de Huancayo, obra que contó con un presupuesto de más de 7 millones de dólares (Andina, 2016).

El Estado puede promover prácticas socialmente responsables a través de exoneraciones fiscales y bonificaciones para aquellas organizaciones que obtienen resultados satisfactorios. También, puede otorgar el financiamiento para programas de desarrollo de estas prácticas. No debe pensarse en una responsabilidad social limitada únicamente a quienes deben llevarla a cabo, el Estado tiene un rol fundamental y en este sentido puede implantar un marco referencial mínimo para la promoción y estímulo de acciones responsables (Soto et al. 2020:2)

En los últimos años las instituciones públicas del Perú se han visto opacadas por las constantes denuncias de corrupción y a pesar de estar vigente desde el año 2002 la Ley № 27658, Ley Marco de Modernización de la Gestión del Estado, se puede evidenciar que los gobiernos locales no desarrollan su labor con responsabilidad social, sobre todo en los diferentes sistemas administrativos de la gestión pública, y peor aún, sin desarrollar a cabalidad el enfoque por resultados.

\section{Conclusiones}

Las organizaciones deben trabajar arduamente en su responsabilidad social para que puedan gestionar de manera sostenible y ética sus relaciones con el ámbito interno y externo y por ende lograr la satisfacción de los empleados. 
Las variables de responsabilidad social y compromiso organizacional en los servidores públicos de la Municipalidad Provincial de Huancayo no tienen relación, es decir, ninguna influye directamente a la otra. A partir de esta investigación se debería implementar un programa de responsabilidad social enfocado en la ISO 26000 es de valiosa importancia en una entidad pública basado en los valores y principios éticos, protección del medio ambiente, proveedores, marketing responsable, apoyo a la comunidad y gobierno y sociedad, el cual permitirá desarrollar las buenas prácticas de gestión pública en cuanto a la rendición de cuentas, la transparencia, al comportamiento ético, al respeto a las partes interesadas y al respeto al principio de legalidad según se dilucido en la teoría del trabajo.

Igualmente, los autores señalaron que la norma ISO 26000 tiende a convertirse en una poderosa herramienta para ayudar a las empresas, gobiernos, asociaciones, entidadesy organizaciones no gubernamentales para incorporar la responsabilidad social como un principio de buena gestión, contribuyendo así a un uso más consciente y sostenible de los recursos naturales y humanos. Es un estándar único, norma voluntaria.

Existen siete principios básicos de la ISO 26000: Rendición de cuentas, transparencia, comportamiento ético, respeto a los intereses de las partes interesadas, respeto del principio de legalidad, respeto a la normativa internacional de comportamiento y respeto a los derechos humanos.

Por otro lado, si bien es cierto la responsabilidad social es compartida con los stakeholders de cualquier organización o institución, sin embargo, existe la falta de sostenibilidad en los procesos, la débil respuesta a las demandas reales de los contextos y la utilización de las acciones de responsabilidad social como mecanismo de represión y control para beneficios particulares han impedido que los esfuerzos que se adelantan en esta materia se traduzcan en verdadero progreso y desarrollo social.

Respecto a la variable compromiso organizacional, la situación es similar, es decir, en base en la teoría estudiada se puede concluir que los servidores públicos no se sienten orgullosos de pertenecer a la Municipalidad porque no han creado lazos emocionales, ni mucho menos disfrutan de su permanencia, tampoco, se sienten vinculados con la institución ya que podrían conseguir otras oportunidades laborales, por más que hayan invertido tiempo, dinero y esfuerzo. Pero contrariamente, hay relación entre la variable responsabilidad social y la dimensión compromiso normativo que significa que se sienten obligados moralmente a pertenecer a la entidad por haberles dado una oportunidad.

Es una realidad que se observa con mayor incidencia en las instituciones públicas el bajo nivel de compromiso organizacional debido a que los servidores públicos son designados o ingresan a laborar como retribución al apoyo que le dieron a la organización política en la campaña política. En los últimos años en la administración pública ha existido favoritismo tanto político como de intereses familiares donde algunos malos servidores públicos colocaban en puestos claves a parientes con la finalidad de lucrar a costa del presupuesto del tesoro público.

Para frenar estos malos manejos se promulgó en Perú la Ley de Nepotismo $\mathrm{n}^{\circ} 26771$, el mismo que establece que los funcionarios de las entidades $y$ 
Responsabilidad social y compromiso organizacional de empleados públicos del Perú

reparticiones públicas que gozan de la facultad de nombramiento y contratación de personal, o tengan injerencia directa o indirecta en el proceso de selección se encuentran prohibidos de ejercer dicha facultad en su entidad respecto a sus parientes hasta cuarto grado de consanguinidad, segundo de afinidad y por razón de matrimonio.

\section{Referencias bibliográficas}

Agencia Peruana de Noticias (27 de noviembre 2016). Identifican presunta responsabilidad penal en exfuncionarios del municipio de Huancayo, Lima, Perú. https://andina. pe/agencia/noticia-identificanpresunta-responsabilidad-penalexfuncionarios-del-municipiohuancayo-823154.aspx

Al-Haroon, Hind y Al-Qahtani, Mona (2020). Assessment of Organizational Commitment Among Nurses in a Major Public Hospital in Saudi Arabia. Journal of Multidisciplinary Healthcare, 13, 519-526. https:// www.magonlinelibrary.com/doi/ pdf/10.12968/bjhc.2018.24.12.603

Artigas, P. (2012). Responsabilidad social enmarcada en los valores del evangelio caso: red de supermercados Sucasa, S.A. (Tesis de Maestría). Universidad Nacional Experimental "Simón Rodríguez", Caracas, República Bolivariana de Venezuela.

Asociación Española de Contabilidad y Administración (2006). La semántica de la responsabilidad social corporativa. Madrid, Gráficas ORMAG, pp.33-40. https://aeca.es/ wp-content/uploads/2014/05/rs3.pdf.

Borthiry Loreficchi, N. (2002). Gestión pública municipal del turismo. Un desafío basado en el fortalecimiento institucional y en la participación.
(Monografía de Graduación), Universidad Nacional de Mar del Plata. http://nulan.mdp.edu. ar/1115/1/borthiry n.pdf, 15/03/2021

Canessa, G., y García, E. (2005). El $A B C$ de la Responsabilidad Social Empresarial en el Perú y en el Mundo. Lima: Siklos S. R. Ltda.

Cano, E., y Vélez, X. (2016). Los diferentes tipos de responsabilidad social y sus implicaciones éticas. Revista científica dominio de las ciencias, 1(2), 117-126. https://dialnet.unirioja.es/servlet/ articulo?codigo $=5802930$

Canyelles, J. (2011). Responsabilidad social de las administraciones públicas. Revista de contabilidad y dirección, 1(13), 77-104. https:// newsletter.collaboratio.net/mm/File/ es/Art.jmcanyelles Nota tecnica Responsabilidad_social de las administraciones publicas RSA.pdf 15/01/2020.

Castillo, F. (2007). Análisis de la responsabilidad social ejercida por el Centro de Entrenamiento Pesquero de Paita: Propuesta de desarrollo. Pontificia Universidad Católica del Perú (tesis), pp 1 - 259.

Chávez, J. C. (2019). Responsabilidad social y compromiso organizacional de los trabajadores de DACIA S.A.C., Comas. (Tesis), Universidad Cesar Vallejo, Lima - Perú. https://hdl. handle.net/20.500.12692/45933

Congreso de la República (1997). Ley de nepotismo $n{ }^{\circ}$ 26771. Establecen prohibición de ejercer la facultad de nombramiento y contratación de personal en el sector público, en casos de parentesco. https://www. oas.org/juridico/PDFs/mesicic4_per ley26771.pdf

Congreso de la República (2020). Ley Marco de Modernización de la Gestión 
del Estado $N^{\circ}$ 27658. https://www. minagri.gob.pe/portal/download/pdf/ cetsar/ley-modernizacion.pdf

Cornejo, A. (2014). Evolución de la RSE en el Perú. Perú 2021, 1(1), 52-54. http://utilidades. gatovolador.net/issuu/down. php?url=https $\% 3 \mathrm{~A} \% 2 \mathrm{~F} \% 2 \mathrm{Fissuu}$. com\%2Fstakeholdersrs\%2Fdocs \%2Fperu 2021 - 20 a os de compromiso \&inicial $=1 \& n p=76$

Đorđević, B., Ivanović, M., Lepojević, V., y Milanović, S. (2020). The impact of employees' commitment on organizational performances. Strategic Management, 25(3), 028$037 . \quad$ http://www.ef.uns.ac.rs/sm/ archive/2020-3/03-SM2020-3.pdf

Ferrer, M. A. (2013). La dimensión externa de la responsabilidad social empresarial de CANTV. Telos, 15(3). http://ojs.urbe.edu/index.php/telos/ article/view/2185

Gaete, R. (2008). Aplicaciones de la Responsabilidad Social a la nueva gestión pública. Documentos $y$ aportes en administración pública y gestión estatal, 1(11), 35-61. https://dialnet.unirioja.es/servlet/ articulo?codigo $=3993367$

Hernández, G. Y. y Castro, D. (2015). Responsabilidad social como estrategia activadora del compromiso organizacional de los trabajadores. Revista Científica Electrónica de Ciencias Gerenciales, 10(1), 123$141 . \quad$ https://www.redalyc.org/ pdf/782/78238677008.pdf

Instituto de Normas Técnicas de Costa Rica, INTECO (2010), Guía de responsabilidad social INTE/ISO 26000:2010. San José: INTECO. https://ucipfg.com/ Repositorio/GSPM/pcMAPV03/ CursosBases/Virtuales/MAPD11-Responsabilidad/Material/ UNDAD2/26000-2010.pdf
Instituto Nacional de Estadística e informática INEI (2017). Junín, Compendio estadístico. $1^{\circ}$ Edición, Instituto Nacional de Estadística e Informática. https://www.inei. gob.pe/media/MenuRecursivo/ publicaciones digitales/Est/Lib1497/ libro.pdf

La Republica (2019). Huancayo: alcalde Chamorro declaró ante Fiscalía Anticorrupción por la Plaza Constitución, Lima, Perú. https:// larepublica.pe/sociedad/1290324huancayo-alcides-chamorro-declarofiscalia-denuncia-delito-peculadomalversacion-plaza-constitucionfuncionarios/?ref=Ire

López, E. (2018). Responsabilidad social y compromiso organizacional de los empleados públicos de la Municipalidad Provincial de Huancayo. (Tesis de Maestría), Universidad Nacional del Centro del Perú, Huancayo, Perú.

Luque, A., Merino, V., y Solís, P. (2019). Gestión pública socialmente responsable: Caso hilando el desarrollo de Ecuador. Revista Venezolana De Gerencia RVG, 24(2), 285-307. https://www. produccioncientificaluz.org/index. php/rvg/article/view/31494/32577

Meyer, J., y Allen, N. (1991). A three component conceptualization of organizational commitment. Human Resource Manegement Review, 1(1), 61-89. https://doi.org/10.1016/10534822(91)90011-Z

Municipalidad Distrital de La Victoria en el Perú (2019). Ordenanza N 006-2019-CPPAL-CDSMC/MDLV. Normas legales - El Peruano. Lima Perú.

Omar, A., y Florencia, A. (2009). Valores personales y compromiso organizacional. Enseñanza e Investigación en Psicología, 1(1), 


\section{López Armes, Edy Luisa}

Responsabilidad social y compromiso organizacional de empleados públicos del Perú

353-372. https://www.redalyc.org/ pdf/292/29213210.pdf

Sánchez, H., Reyes, C., y Mejía, K. (2018). Manual de términos en investigación científica, tecnológica y humanística. Trabajo académico, Universidad Ricardo Palma, Perú. http://repositorio.urp.edu.pe/handle/ URP/1480?show=full

Soto, L., y Batista, J. (2020). La responsabilidad social de la empresa estatal en el ejercicio pleno de los derechos de los trabajadores en la empresa estatal en Cuba. Revista IUS, 1(45), 55-79. http://www.scielo. org. $\mathrm{mx} / \mathrm{pdf} / \mathrm{rius} / \mathrm{v} 14 \mathrm{n} 45 / 1870-2147$ rius-14-45-55.pdf
Tomba, C., Muñoz, C. y Allisiardi, A. (2020). La responsabilidad social de la publicidad: el rol de las campañas de bien público. Revista VivatAcademia, XIII (150), 103129. https://www.vivatacademia.net/ index.php/vivat/article/view/1190

Vargas-Merino, J. A. (2021). Innovación social: ¿Nueva cara de la responsabilidad social? conceptualización crítica desde la perspectiva universitaria. Revista De Ciencias Sociales, 27(2), 435450. https://doi.org/10.31876/rcs. v27i2.35934 